\title{
Slit beam shaping technique for femtosecond laser inscription of enhanced plane-by-plane FBGs
}

\author{
Pablo Roldán-Varona, David Pallarés-Aldeiturriaga, Luis Rodríguez-Cobo, and José Miguel López-Higuera
}

\begin{abstract}
In this work, the development of plane-by-plane (Pl-b-PI) fiber Bragg gratings (FBGs) by using a single pulse is reported. A slit oriented along the longitudinal axis of the fiber is employed for this. The purpose is to shape the beam so that the focal volume is spatially wider in the transverse direction of the fiber. In this way, it is possible to make 2-D modifications of the refractive index whose width and height depends on the slit width and the pulse energy, respectively. Through an analytical mathematical modelling, the relationship between the diameter of the Gaussian laser beam $\left(D_{x}\right)$ and the slit width $\left(s=D_{y}\right)$ is determined in order to obtain a circular crosssection. In the experimental results carried out, it can be observed that $D_{x} / D_{y}=3$ ratio cause positive and uniform refractive index changes, compared to the negative and inhomogeneous refractive index changes corresponding to point-by-point (PbP) FBGs. Likewise, PI-b-PI FBGs, presenting a broader modification of the core cross-section, show significantly lower losses in transmission $(0.3 \mathrm{~dB})$, as well as better reflectivity and FWHM. It is observed that these three parameters (losses, reflectivity and FWHM) have a monotonous tendency according to the slit width. Polarizationdependence is also evaluated.
\end{abstract}

Index Terms-Femtosecond laser, Optical Fiber Sensor, Slit beam technique, Fiber Bragg grating, Plane-by-Plane.

\section{INTRODUCTION}

$\mathbf{F}$ IBER Bragg gratings (FBGs) written with femtosecond (fs) lasers exhibit a great interest given the increased temperature resistance and also a better flexibility of wavelength and composition, thanks to their characteristic high peak power densities that makes possible to achieve nonlinear ionization at arbitrary locations [1,2]. Besides, FBGs can not only be manufactured with the phase mask technique [3], but also can be produced with the direct-write technique, which is far more versatile than phase mask (works only for a given period and wavelength), being attractive for multiplexing applications [4].

Within the direct-write technique [5], there are three types of inscription, namely, point-by-point (PbP), line-by-line ( $\mathrm{LbL}$ ) and plane-by-plane (Pl-b-Pl). The first one, $\mathrm{PbP}$, essentially

This work has been supported by the project TEC2016-76021-C2-2-R (AEI/FEDER) of the Spanish government and FEDER funds, and by the grant FPU2018/02797 from the Ministry of Science, Innovation and Universities of the Spanish government. (Corresponding author: Pablo Roldán-Varona)

P. Roldán-Varona is with the Photonics Engineering Group, University of Cantabria, 39005, Santander, Spain; and Instituto de Investigación Sanitaria Valdecilla (IDIVAL), Cantabria, Spain (e-mail: pablo.roldan@unican.es).

D. Pallarés-Aldeiturriaga is with the Photonics Engineering Group, University of Cantabria, 39005, Santander, Spain; and the CIBER-bbn, Instituto de Salud Carlos III, 28029, Madrid, Spain (e-mail: david.pallares@unican.es).

L. Rodríguez-Cobo is with the CIBER-bbn, Instituto de Salud Carlos III, 28029, Madrid, Spain (e-mail: luis.rodriguez@unican.es).

J.M. López-Higuera is with the Photonics Engineering Group, University of Cantabria, 39005, Santander, Spain; the CIBER-bbn, Instituto de Salud Carlos III, 28029, Madrid, Spain; and Instituto de Investigación Sanitaria Valdecilla (IDIVAL), Cantabria, Spain (e-mail: miguel.lopezhiguera@unican.es). consists in the inscription within the fiber core of a line parallel to the fiber axis. The line is inscribed with a writing speed $v=\Lambda \cdot$ PRR were $\Lambda$ is the grating period and PRR the pulse repetition rate [6], allowing single pulses to be deposited with proper separation (Figure 1 - PbP). This type of inscription is simple and straightforward. However, it usually exhibits strong broadband losses $[7,8]$, lower grating strength and also higher order modes coupling. To limit the latter, any refractive index change must precisely coincide with the longitudinal axis of the optical fiber, requiring precise alignment. This can be explained through the Coupled Mode Theory (CMT) and the transversal coupling constant between two modes [9]:

$$
\kappa_{a b}=\iint 2 \epsilon_{0} \Delta n_{m}(x, y) n(x, y) \mathbf{E}_{\mathbf{a}}^{\mathbf{T}}(x, y) \mathbf{E}_{\mathbf{b}}^{\mathbf{T} *}(x, y) \cdot \mathrm{d} x \mathrm{~d} y .
$$

Here, if $\Delta n_{m}(x, y)$ covers the entire cross-section of the fiber homogeneously (being constant), then the orthogonality relation will exclude coupling between different modes. However, if $\Delta n_{m}(x, y)$ is asymmetric as the laser modification does not cover the entire core cross-section, then light will be decoupled to higher $m$ and $l$ modes [6].

The LbL technique was first employed by Zhou et al. [10] and is depicted in Figure 1 - LbL. Here, instead of a single inscription, several lines are inscribed perpendicular to the longitudinal axis. This method is significantly slower than $\mathrm{PbP}$, but covers more cross-section of the core, reducing the amount of cladding modes coupled. However, the line geometry is quite exploited to manufacture tilted FBGs where cladding modes are enhanced, allowing sensitivity to external changes. This larger and also smoother area can allow lower broadband losses, which depend on the length and roughness of the lines.. Furthermore, the LbL inscription method allows the manufacture of relatively complex structures, by allowing flexible control over each inscribed line (e.g. induced refractive index change [11]).

Finally, the Pl-b-Pl method involves the inscription of a plane that produces a quasi-homogeneous $2 \mathrm{D}$ refractive index change in the core (or cladding) cross-section. This method involves flexible control of the width (direction of the $Y$ axis in Fig. 1) and height ( $Z$ axis) of the planes, since the thickness of the plane ( $X$ axis) is defined by the laser wavelength and the numerical aperture of the objective lens. Thus, width and height of equal dimensions are possible. Plb-Pl method, depicted in Figure 1 - Pl-b-Pl, was first reported in 2016 by Theodosiou et al. [4], and has subsequently been used in multiple applications, such as polymer optical fibers [12], or the inscription of gratings and interferometric in-fiber components $[13,14]$. Another attractive way to achieve such 


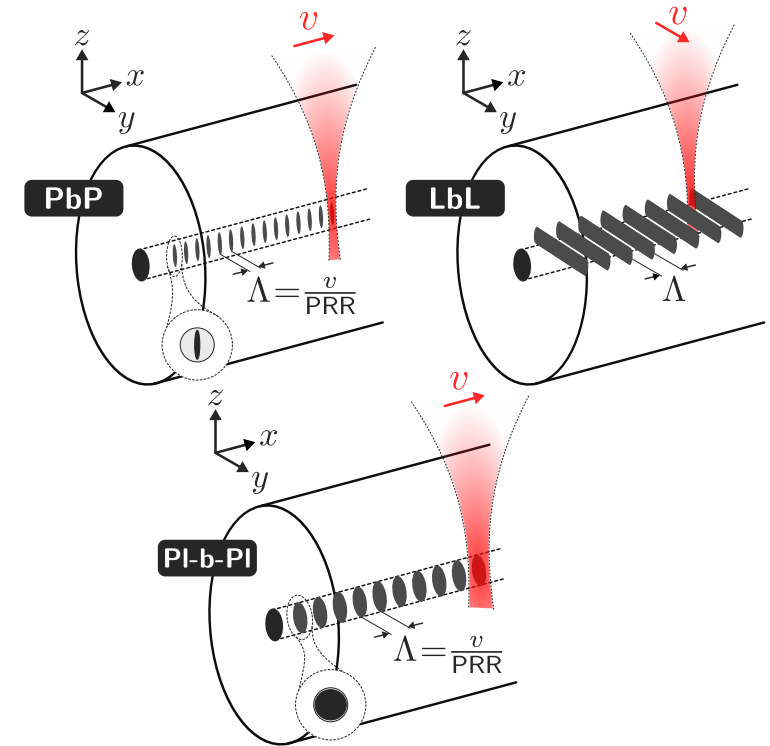

Fig. 1. Methods of FBG inscription with fs laser (PbP, LbL, Pl-b-Pl)

control is by shaping the focal volume into a plane, thus writing planes in a single pulse in a similar way to $\mathrm{PbP}$ method. This was successfully achieved by $L u$ et al. by means of a cylindrical lens before the microscope objective, which induces astigmatism in the focal volume [15]. This concept is quite similar to the astigmatic beam shaping technique, where a cylindrical telescope modifies the resulting Rayleigh range after the microscope lens in order to achieve circular waveguides [16]. Another method to get planar modifications with a single pulse is the slit beam method, employed by Ams et al. to carry out waveguides [17]. Here, the cylindrical telescope is replaced by a a simple slit before the objective lens, being more compact and requiring much less adjustment , but with a higher pulse energy requirement. The use of a slit was also used to reduce the focal volume distortions caused by the induced astigmatism due to the cylindrical fiber geometry [18].

In this work the aforementioned methodology will be employed to generate Pl-b-Pl gratings with a single pulse. Here, one step further is taken by shaping the beam to a more adequate geometry in order to achieve higher quality inscription. For such purpose, a theoretical revision will be performed to select the most adequate slit width for the current setup. Then, the inscribed gratings will be characterized using quantitative phase microscopy (QPM) to compare geometry and refractive index change of the new Pl-b-Pl gratings with their $\mathrm{PbP}$ counterpart. Finally,the transmission and reflection spectra are presented, as well as the polarization dependent loss, in order to study the improvements achieved with this new method.

\section{SLIT BEAM TEChNiQUE}

As mentioned earlier, there are multiple ways to control the cross-section of the focal volume, through a variation in the beam waist ratio. However, most of them require additional optics, such as astigmatic beam shaping [16], active optics
[19] or spatiotemporal focussing (STF) techniques [20], so alignment can be a major problem.

The slit beam technique consists in modifying the beam previously to the objective lens by introducing a slit, which offers greater simplicity and control than previous techniques. It should be noted that the focused beam waist on each axis depends on the numerical aperture (NA) on each axis, the NA being dependent on both the focal length of the objective lens $(f)$ and the diameter of the beam on each axis $\left(D_{x}, D_{y}\right)$.

$$
w_{0 i}=\frac{\lambda}{\pi \mathrm{NA}_{i}} \simeq \frac{\lambda}{\pi} \frac{f}{D_{i}}, \quad \forall i=\{x, y\} .
$$

According to the spatial arrangement of axes shown in Figure 1 , if it is necessary to look for a planar modification of the fiber core, the focussed beam waist of the $Y$ axis $\left(w_{0 y}\right)$ must be increased. Because of this, the NA of that axis and, therefore, the diameter of the beam in said axis $\left(D_{y}\right)$ has to be reduced. Consequently, the slit is oriented along the $X$ axis, and its width $(s)$ defines $D_{y}$.

After the slit, an elliptic Gaussian beam can be assumed, in which the resulting diffractive effect of the slit causes a redistribution of optical energy around the focus [21]. However, to assume an elliptical Gaussian beam at the input of the objective lens, the distance between the slit and the objective lens must always be less than $d_{R}=s^{2} / \lambda$ (Rayleigh range), with $s$ the slit width. In this way, operating in the nearfield regime, the Fraunhofer diffraction can be neglected. In order to simplify the calculation, regardless of this effect, the energy distribution around the focal volume can be expressed as:

$$
I(x, y, z)=\frac{I_{0} \cdot w_{0 x} w_{0 y}}{w_{x}(z) w_{y}(z)} e^{-2\left[\left(\frac{x}{w_{x}(z)}\right)^{2}+\left(\frac{y}{w_{y}(z)}\right)^{2}\right]},
$$

with

$w_{i}(z)=w_{0 i} \sqrt{1+\left(\frac{z}{z_{0 i}}\right)^{2}}, \quad z_{0 i}=\frac{n \lambda}{\pi \mathrm{NA}_{i}^{2}}, \quad \forall i=\{x, y\}$,

where $z_{0}$ is the Rayleigh length, and $n$ is the refractive index of the substrate. Then, applying a paraxial approach, and assuming that the beam that reaches the slit (located before the objective lens) is circular Gaussian, an analytical development is carried out that aims to define the $D_{x} / D_{y}$ relationships that cause a circular cross-section in the $Y Z$ plane. Unlike [17], no starting assumption will be made regarding the relationship between $D_{x}$ and $D_{y}$.

Let $y_{I / 2}$ be the distance on the $Y$ axis (with respect to the focal point) at which the intensity drops by a factor 2 , and $z_{I / 2}$ the equivalent on the $Z$ axis, the cross-section will be circular when $y_{I / 2}=z_{I / 2}$ (half-width-half-maximum approach). Starting from Eq. 2 the development is carried out:

- $Y$ AXIS $(x=0, z=0)$

$$
\begin{aligned}
I\left(0, y_{I / 2}, 0\right) & =\frac{I_{0}}{2}=I_{0} \cdot e^{\frac{-2 y_{I / 2}^{2}}{w_{0 y}^{2}}} . \\
y_{I / 2} & =w_{0 y} \sqrt{\frac{\ln (2)}{2}} .
\end{aligned}
$$


- $Z$ AXIS $(x=0, y=0)$

$$
\begin{gathered}
I\left(0,0, z_{I / 2}\right)=\frac{I_{0}}{2}=I_{0} \frac{1}{\sqrt{1+\left(\frac{z_{I / 2}}{z_{0 x}}\right)^{2}} \sqrt{1+\left(\frac{z_{I / 2}}{z_{0 y}}\right)^{2}}} . \\
\left(z_{I / 2}^{2}+z_{0 x}^{2}\right)\left(z_{I / 2}^{2}+z_{0 y}^{2}\right)=4 z_{0 x}^{2} z_{0 y}^{2} .
\end{gathered}
$$

Equating $y_{I / 2}$ and $z_{I / 2}$ of Equation 3 and Equation 4, respectively, gives the following expression, dependent on $D_{x}$ and $D_{y}$. The results obtained are shown in Figure 2, compared to the results given in [17], which make the assumption that $D_{x}>3 D_{y}$.

$$
\left(1+\alpha \frac{D_{x}^{4}}{D_{y}^{2}}\right)\left(1+\alpha D_{y}^{2}\right)=4, \quad \text { with } \quad \alpha=\frac{\ln (2)}{2 n^{2} f^{2}}
$$

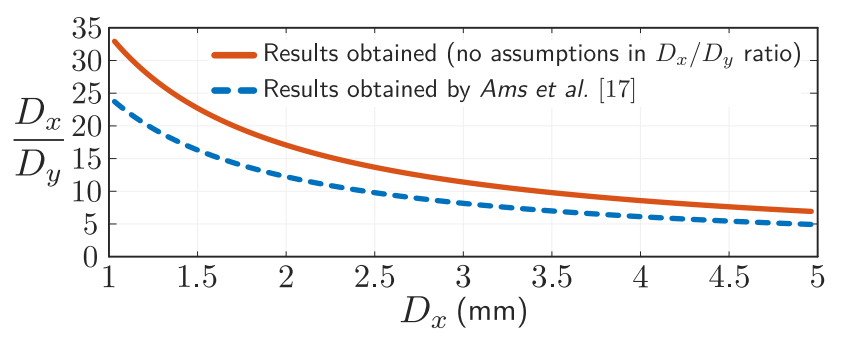

Fig. 2. Relationship between $D_{x}$ and $D_{y}$ as a function of $D_{x}$ to obtain a circular cross-section of the focal volume in the $Y Z$ plane. A substrate with $n=1.47$ and a focal length of the objective lens $f=8 \mathrm{~mm}$ has been used.

The results obtained can be adjusted to the expression shown below, which offers a coefficient of determination $R^{2}=0.9994$ for values of $D_{x}$ between 1 and $12 \mathrm{~mm}$.

$$
\frac{D_{x}}{D_{y}} \simeq 33.97 \cdot\left[D_{x}(\mathrm{~mm})\right]^{-0.993}
$$

It should be noted that, through the use of the slit, the symmetry of the cross-section can be significantly improved, but at the cost of a decrease in efficiency, since part of the initial pulse energy is lost. This trade-off implies that, sometimes (as it happens in this work), it is not interesting to get a circular cross-section if this implies a very reduced efficiency. There are some alternatives that, although they require a complex optical alignment, allow to overcome this issue $[16,22]$.

Next, based on simulations performed, the $Y Z$ plane of the focal volume for different $D_{x} / D_{y}$ ratios is presented (Figure 3), depicting the variations of its ellipticity. The circular cross-section is obtained for $D_{x} / D_{y}=12$, which corresponds to the result shown in Figure 2 for $D_{x}=3 \mathrm{~mm}$.

Finally, the effect related to efficiency is shown. Figure 4 indicates the evolution of the intensity along the fiber (focusing the core) with $x=y=0$. It is observed that a ratio $D_{x} / D_{y}=$ 2 causes a reduction in intensity at the focal point to $28 \%$ compared to the case without slit. If a circular cross-section is required $\left(D_{x} / D_{y}=12\right)$, the energy at the focal point is only $0.8 \%$ compared to the case without slit. That is why getting a circular cross-section causes a significantly higher laser pulse energy (since the slit blocks most of the power).

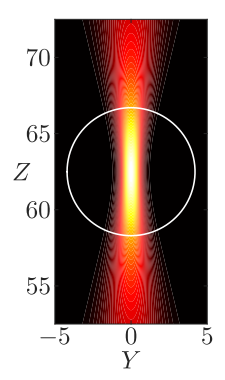

(a)

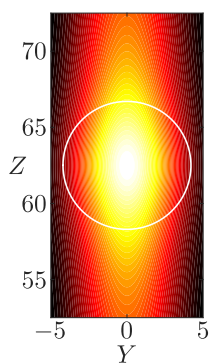

(b)

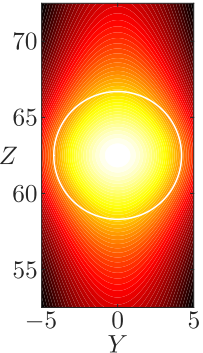

(c)

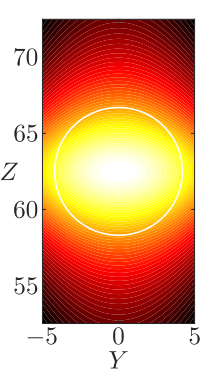

(d)
Fig. 3. $Y Z$ plane of the focal volume for $D_{x} / D_{y}$ ratios of (a) 1 , (b) 6 , (c) 12 and (d) 20. The white circle represents the core of the optical fiber. A substrate with $n=1.47$ and a focal length of the objective lens $f=8 \mathrm{~mm}$ has been used.

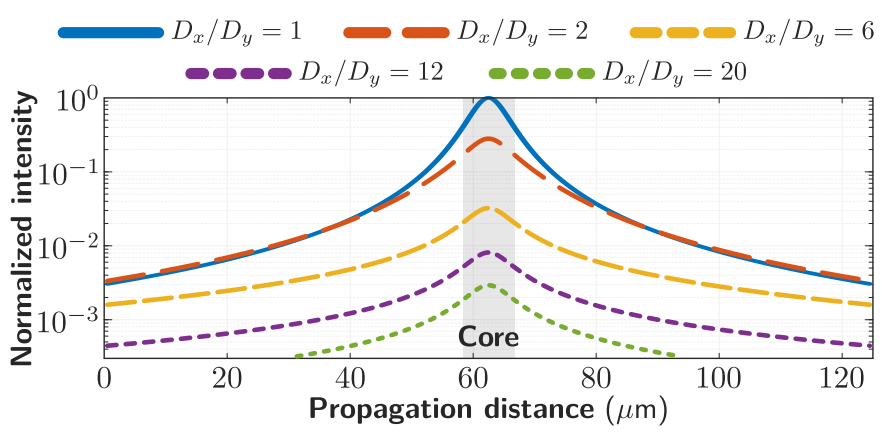

Fig. 4. Evolution of intensity on the $Z$ axis $(x=y=0)$ with focus on the center of the SMF core $(z=62.5 \mu \mathrm{m})$. Different $D_{x} / D_{y}$ ratios are depicted.

\section{SETUP AND INSCRIPTION PARAMETERS}

FBG inscriptions have been made using a commercial femtosecond Fiber Laser Chirp Pulse Amplifier (FLCPA) from CALMAR laser, operating at $1030 \mathrm{~nm}$, with a $370 \mathrm{fs}$ pulse duration and a variable PRR from $1 \mathrm{~Hz}$ to $120 \mathrm{kHz}$. As it is depicted in Figure 5, laser pulses pass through an iris diaphragm $(\varnothing 3 \mathrm{~mm})$ in order to provide a clean wavefront, and then go through the adjustable slit, whose purpose is the one discussed in the previous section. The slit is oriented along the $\mathrm{X}$ axis, the inscription direction.

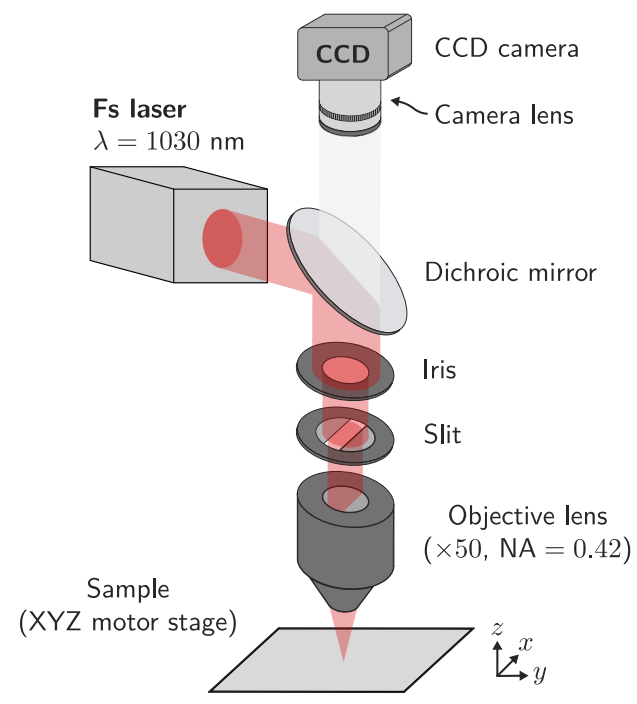

Fig. 5. Manufacturing stage. Schematic setup for the FBG inscription. 
Subsequently, the pulses are focused using an objective lens from Mitutoyo with NA $=0.42$ and a $\times 50$ magnification. The strechted optical fiber is located on a slide and covered with a coverslip, and between them there is an index-matching oil that allows to avoid the aberration inherent to the fiber [23]. The sample is placed on a nanoresolution XYZ motor stage from Aerotech. Finally, by means of a transmission illumination and a CCD camera, it is possible to position and visualize the fiber correctly.

\section{A. Pulse energy setting}

The main consideration to take into account is that the incident pulse energy (at the laser output) is not the same as that which reaches the sample. Effective pulse energy $\left(\left(E_{p}\right)_{e f f}\right)$ will be discussed to refer to the energy that passes through the slit. In order to compare the inscribed structures by varying the width of the slit, $\left(E_{p}\right)_{\text {eff }}$ must be kept constant. In this way, the induced laser energy in the focal volume is not modified. Considering that the incident pulse energy follows a Gaussian spatial distribution $\left(M^{2}<1.1\right)$

$$
E(x, y)=A \cdot e^{\left[-\left(\frac{x^{2}}{2 \sigma_{x}^{2}}+\frac{y^{2}}{2 \sigma_{y}^{2}}\right)\right]},
$$

with a beam diameter $2.5 \mathrm{~mm}\left(1 / e^{2}\right)$, it is important to indicate the percentage of light that passes through the slit based on its width $(s)$, and determine the pulse energy to be used with each value of $s$ so that $\left(E_{p}\right)_{\text {eff }}$ is always the same.

The effective pulse energy with which the FBGs will be inscribed will be $2.84 \mu \mathrm{J}$. Figure 6 shows the necessary incident pulse energy.

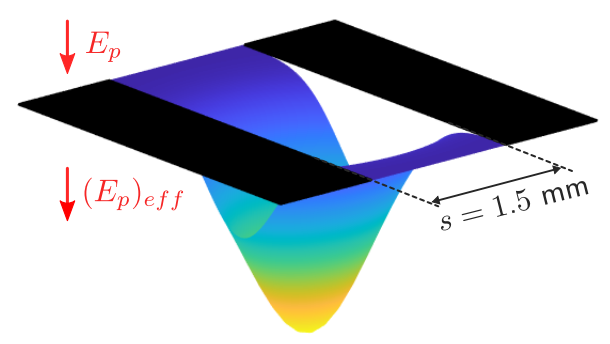

(a)

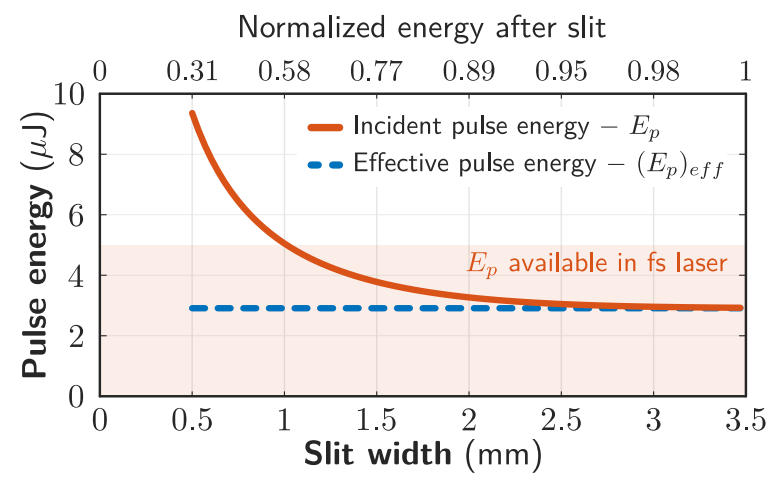

(b)

Fig. 6. (a) Example of evolution of a laser pulse through a $1.5 \mathrm{~mm}$ width slit, with the definition of $E_{p}$ and $\left(E_{p}\right)_{e f f}$. (b) Variation of $E_{p}$ depending on the slit width to maintain a constant $\left(E_{p}\right)_{\text {eff } f}$.

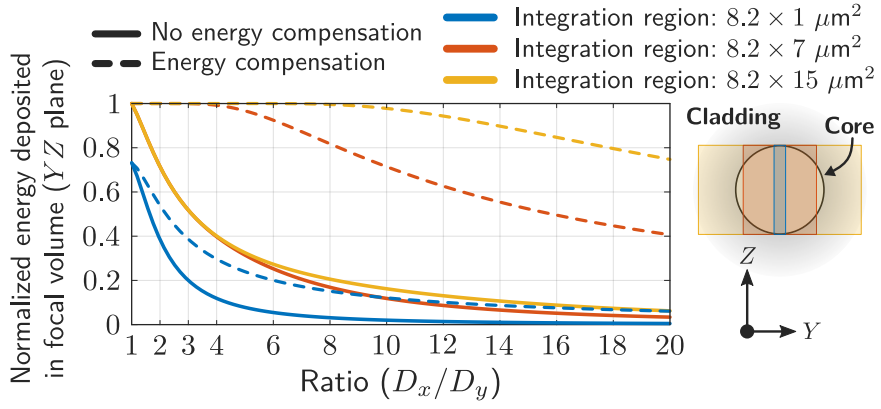

(a)

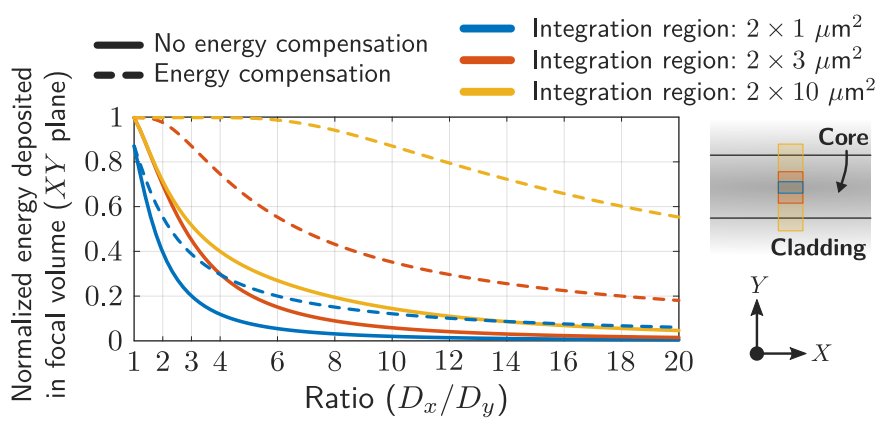

(b)

Fig. 7. Normalized energy deposited in the focal volume, considering (a) the $Y Z(x=0)$, (b) and $X Y(z=0)$ planes. The $Y Z$ plane corresponds to the core cross-section, while the $X Y$ plane represents a longitudinal section of the fiber. The results are depicted both without energy compensation (solid line), and with the energy compensation detailed in this section (dotted line). Different colors refer to different integration regions of energy fluence.

In the case presented in this article, it is intended to compare $\mathrm{PbP}$ and Pl-b-Pl FBGs inscribed with identical energy in the focal volume. In this way, at any ratio $D_{x} / D_{y}$, it is ensured that the energy deposited in the focal volume is practically identical. In Figure 7 it is explicitly depicted that the detailed energy compensation (effective pulse energy) allows the same energy to be available in the focal volume. Fluence is integrated into the integration regions shown, both in the $Y Z$ (Figure 7a) and $X Y$ (Figure 7b) plane. In both cases, the comparison of the case without energy compensation (same energy at the laser output) or with energy compensation is shown. Smaller focal volumes imply higher fluence in $\mathrm{PbP}$, while Plb-Pl focal volumes contain lower and smoother fluence. This is why small integration regions cause a greater amount of energy in $\mathrm{PbP}$ than in Pl-b-Pl. However, in both cases the energy deposited in the focal volume can be considered the same. For higher ratios, the integration region should be expanded.

Another interesting technique would be to equalize the energy fluence at the center of the focal volume. Obviously, this requires increasing the energy deposited in focal volume as the $D_{x} / D_{y}$ increases. However, even if the intensity of the focal points is equalized, the smaller width of focal volume would cause a more localized stress at core and lower grating strength in $\mathrm{PbP}$.

FBGs will be performed in an SMF-28 core for slit widths $1,1.5,2$ and $3 \mathrm{~mm}$, which requires incident pulse energies of $4.93,3.69,3.19$ and $2.88 \mu \mathrm{J}$, respectively. The laser PRR is set at $30 \mathrm{~Hz}$, and the period of the FBGs takes a value of $\Lambda=2.15 \mu \mathrm{m}$, which generates the 4 th order in the C-band. The writing speed is therefore $v=\Lambda \cdot \operatorname{PRR}=64.5 \mu \mathrm{m} / \mathrm{s}$. 


\section{EXPERIMENTAL RESULTS AND DISCUSSION}

\section{A. QPM characterization}

Once inscribed the aforementioned gratings, a phase study can be performed to contrast with the theoretical results. It should be noted that the refractive index is proportional to the phase $(n \propto \phi)$. Quantitative Phase Microscopy (QPM) is a relatively straightforward method to retrieve phase images from intensity images exploiting the intensity transport equation [24]. The average phase change $(\Delta \phi)$ and width of the gratings depending on the slit width $(s)$ is depicted in Figure 8. Here, first and foremost, the inscription width increases for $D_{x} / D_{y}>1.5$ up to a 3 factor $(s=1 \mathrm{~mm})$, which is almost the core width. Besides, another interesting property of $D_{x} / D_{y}>2$ ratios is its positive phase change compared with higher slit values, where the $\mathrm{PbP}$ counterpart shows higher but negative phase changes. These modifications become more inhomogeneous, as shown in the transversal profile of Figure 9a. In fact, the phase change decreases with an exponential curve $\left(R^{2}=0.9982\right)$. Figure 9a depicts three phase profiles corresponding to a pristine fiber and $s=\{1,3\} \mathrm{mm}$. The $s=1 \mathrm{~mm}$ inscription not only produces a positive phase change as indicated above, but also generates a homogeneous modification compared with $s=3 \mathrm{~mm}$.

The amplitude of this phase change has also been studied. The amplitude for $s=1 \mathrm{~mm}$, as depicted in Figure $9 \mathrm{~b}$ is weak compared with larger slit widths such as 2 and $3 \mathrm{~mm}$, that are approximately equal in amplitude, being $s=2 \mathrm{~mm}$ slightly more sinusoidal. In these $\mathrm{PbP}$ cases, focal volume is more compact, thus, the expansion produced generates higher phase changes. Besides, surrounding area exhibits a stress associated with an index change of opposing sign and without grating modulation. Following Equation 1, a higher index amplitude $\left(\Delta n_{m}\right)$ might also suggest a higher coupling coefficient. However, the lower cross-section and the stress region must also be taken into account. Particularly, the induced index change surrounding the grating can easily induce losses that compromise the ideal transmission and reflection. This will be discussed further in the next section.

All inscriptions correspond to Type I changes in the material. According to the literature, Type II corresponds to a birefringent change in the refractive index, due to the formation of nanogratings in the material. However, and according to multiple proven works $[2,25]$, to achieve such a change,

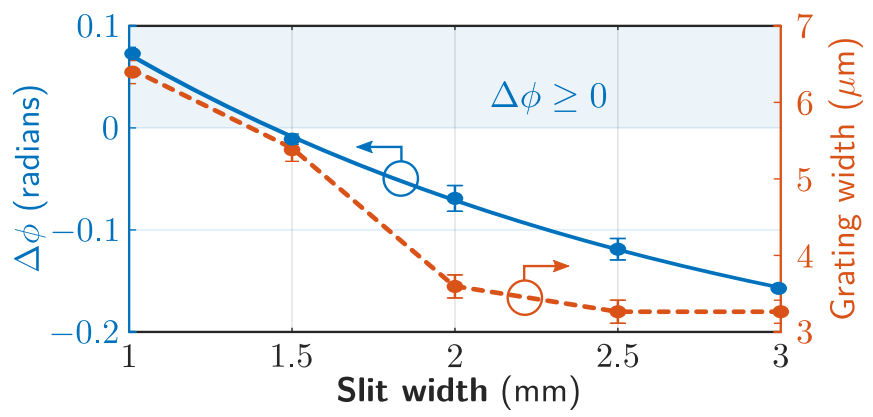

Fig. 8. Average phase change and modification width for different slit widths $(s)$. Phase change decreases according to an exponential fit $\left(R^{2}=0.9982\right)$.

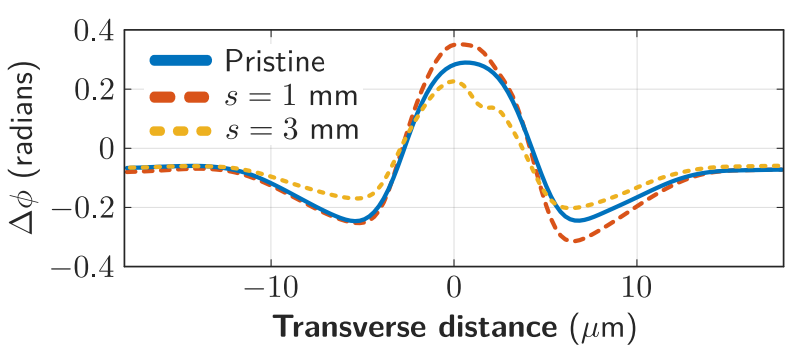

(a)

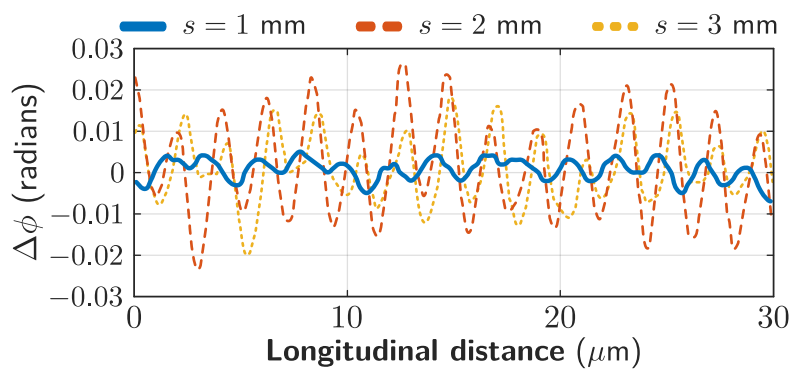

(b)

Fig. 9. (a) Transverse phase profile for pristine and $s=\{1,3\} \mathrm{mm}$, (b) and phase change parallel to grating inscription for $s=\{1,2,3\} \mathrm{mm}$.

the interaction of two or more pulses is required. This is why the existence of nanogratings in the FBGs seems unlikely. In summary, PbP FBG is a more birefringent structure, due to the asymmetry of its focal volume and the more localized stress in the core. On the other hand, in Pl-b-Pl FBGs, although there is also induced stress, anisotropic behavior is markedly inferior. Likewise, the generated planes present a more symmetrical and homogeneous change.

\section{B. Pl-b-Pl FBGs with slit method}

The inscription of Pl-b-Pl FBGs by means of the slit beam technique allows to have a precise control of the grating period, as well as the width and height of the planes, depending on the slit width and the pulse energy, respectively. In this way it is possible to have a certain flexibility in the performance of the optical structure.

FBGs have been characterized with the slit widths and pulse energies shown in subsection III-A. The results of inscription for slit widths of $1 \mathrm{~mm}\left(D_{x} / D_{y} \simeq 3\right)$ and $3 \mathrm{~mm}\left(D_{x} / D_{y} \simeq 1\right)$ are depicted in Figure 10. Since $\left(E_{p}\right)_{e f f}$ is equal, the increase in plane width can be observed when $s$ (slit width) decreases. The PbP FBG is achieved when the width of the slit is similar to the diameter of the laser beam $\left(1 / e^{2}\right)$, that is, $2.5 \mathrm{~mm}$.

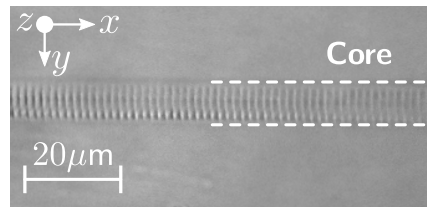

(a)

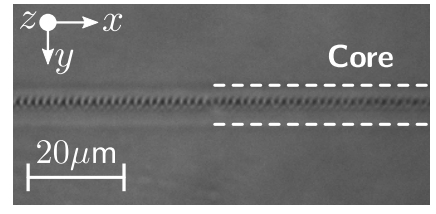

(b)
Fig. 10. FBGs inscribed in the core of an SMF-28 fiber with (a) $s=1 \mathrm{~mm}$ (Pl-b-Pl), (b) and $s=3 \mathrm{~mm}(\mathrm{PbP})$.

The FBGs, with a length $L=1 \mathrm{~mm}$ (inscription time $15 \mathrm{sec} / \mathrm{FBG}$ ), have been characterized both in reflection and 


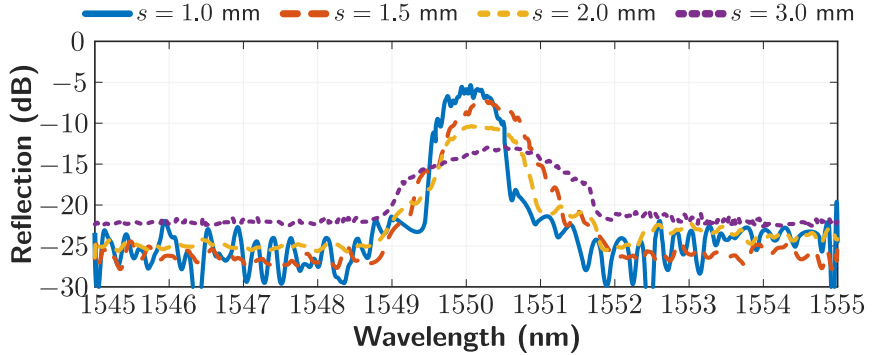

(a)

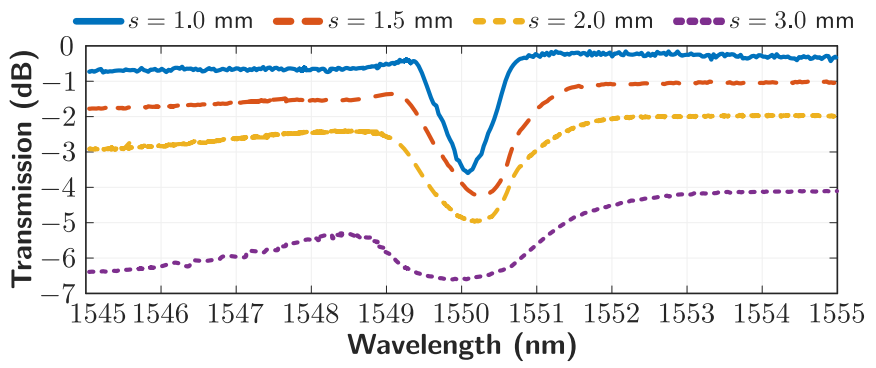

(b)

Fig. 11. (a) Reflection and (b) transmission spectra of FBGs inscribed with $s=\{1,1.5,2,3\} \mathrm{mm}$. These are traces of 2001 points (50-point average).

transmission using a broadband light source (HP 83437A) and an Optical Spectrum Analyzer (Anritsu MS9740A) with $30 \mathrm{pm}$ resolution. Reflection and transmission spectra are depicted in Figure 11 for slit widths $s=\{1,1.5,2,3\} \mathrm{mm}$.

It is noteworthy the increase of broadband losses when the inscription approaches a $\mathrm{PbP}$, exhibiting $4 \mathrm{~dB}$ transmission losses for the $\mathrm{PbP}$ and $0.3 \mathrm{~dB}$ for the $D_{x} / D_{y} \simeq 3 \mathrm{Pl}$-b$\mathrm{Pl}$ inscription (Figure 10a). These losses, that are better than what exists in the literature [10], are consistent with phase results discussed in subsection IV-A, where the stress region and lower cross-section play a major role. This transmission losses slightly increase broadband reflection, being $\mathrm{PbP}(s=3$ $\mathrm{mm})$ reflection approximately $6 \mathrm{~dB}$ higher than Pl-b-Pl $(s=$ $1 \mathrm{~mm}$ ). However, the results presented here are intended to show the tendency by varying the slit width. By choosing the right energies, it is effectively possible to manufacture highperformance FBGs with both $\mathrm{PbP}$ and Pl-b-Pl methods.

In terms of FWHM and reflection, the slit width reduction clearly improves both parameters. These trends are depicted in Figure 12, where FWHM decreases with the increase of $D_{x} / D_{y}$, while reflectivity exhibits a monotonous increment. The reflectivity can reach $-5 \mathrm{~dB}$ and the FWHM $\sim 0.69 \mathrm{~nm}$

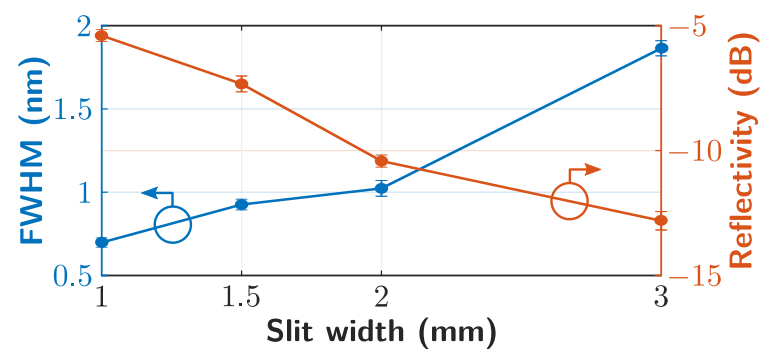

Fig. 12. Dependence of the FWHM and the reflectivity with the slit width. for $s=1 \mathrm{~mm}(\kappa L \approx 0.65$, being $\kappa$ the coupling constant). The increase in reflectivity can be explained thanks to the width increase and the loss reduction that allows more light to cross the entire grating length. Similarly, the FWHM reduction is consistent with lower slit widths where cross-section is not only higher but also homogenous as depicted in Figure $9 b$. In this way, it is clear that inscribed Pl-b-Pl gratings with presented method exhibit a higher quality index modulation which translates into a better response.

In order to verify the results obtained, the polarizationdependece of $\mathrm{PbP}(s=3 \mathrm{~mm})$ and Pl-b-Pl $(s=1 \mathrm{~mm})$ FGBs is evaluated. Determining the maximum change in transmitted (or reflected) power when the input state of polarization is varied over all polarization states is a good way to characterize the structures presented in this work [26]. A polarization controller and a broadband light source have been used to measure the polarization dependence of the FBGs. The results are depicted in Figure 13. The PbP FBG presents a greater variation with the polarization state. This is due to the greater birefringence it presents, as well as the marked transverse asymmetry of each modification. For the opposite reasons, the Pl-b-Pl FBG has a significantly lower PDL $(\sim 1.5 \mathrm{~dB})$. It should be noted that, ideally, in a Pl-b-Pl FBG it would be possible to maintain the cylindrical symmetry of the fiber by pulses that originate circular planes (Figure 2). The Bragg wavelength shift of the different polarization states is always less than $70 \mathrm{pm}$.

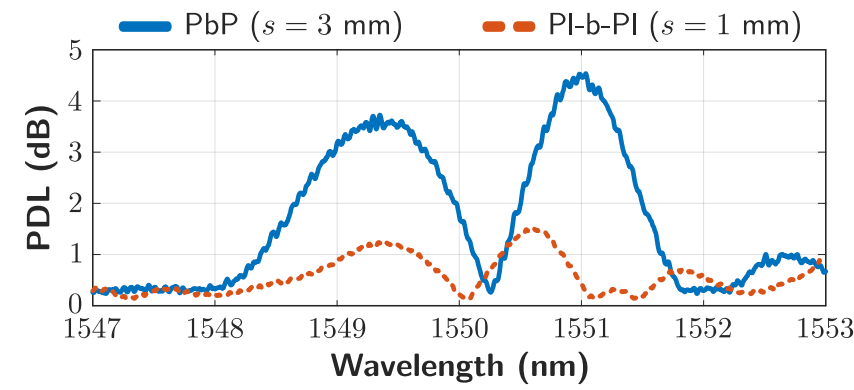

Fig. 13. Polarization dependent loss (PDL) of $\mathrm{PbP}\left(s=3 \mathrm{~mm}, D_{x} / D_{y}=1\right)$ and Pl-b-Pl $\left(s=1 \mathrm{~mm}, D_{x} / D_{y}=3\right)$ FBGs.

\section{CONCLUSION}

A new method of inscription of plane-by-plane FBGs has been presented and verified both theoretically and experimentally. By using a slit located before the objective lens, it is possible to induce quasi-homogeneous two-dimensional refractive index changes (RIC), with flexible control over the width and height of the grating plane. Although the energy efficiency is lower than in other methods, the inscription speed is very high since a plane is induced with each laser pulse. A better spectral response is obtained than through the $\mathrm{PbP}$ and LbL methods, in terms of losses, FWHM and reflectivity. Similarly, Pl-b-Pl FBGs have a very reduced dependence on the polarization state. Likewise, through a phase characterization of these modifications, smooth and uniform changes are observed in Pl-b-Pl FBGs $\left(D_{x} / D_{y}=3\right)$, unlike the negative and inhomogeneous changes of the PbP FBGs, derived from Gaussian laser beams without previous shaping beam. 


\section{REFERENCES}

[1] P. Günter and J. P. Huignard, Photorefractive materials and their applications 2: Materials. Springer, 2007.

[2] F. Dürr, "Laser-induced stress changes in optical fibers," EPFL, Tech. Rep., 2005.

[3] S. Mihailov, C. Smelser, D. Grobnic, R. Walker, P. Lu, H. Ding, and J. Unruh, "Bragg gratings written in All- $\mathrm{SiO}_{2}$ and $\mathrm{Ge}$-doped core fibers with 800-nm femtosecond radiation and a phase mask," Journal of Lightwave Technology, vol. 22, no. 1, pp. 94-100, jan 2004.

[4] A. Theodosiou, A. Lacraz, M. Polis, K. Kalli, M. Tsangari, A. Stassis, and M. Komodromos, "Modified fslaser inscribed fbg array for rapid mode shape capture of free-free vibrating beams," IEEE Photonics Technology Letters, vol. 28, no. 14, pp. 1509-1512, 2016.

[5] A. Martinez, M. Dubov, I. Khrushchev, and I. Bennion, "Direct writing of fibre bragg gratings by femtosecond laser,' Electronics Letters, vol. 40, no. 19, pp. 11701172, 2004.

[6] J. Thomas, C. Voigtlaender, R. G. Becker, D. Richter, A. Tuennermann, and S. Nolte, "Femtosecond pulse written fiber gratings: a new avenue to integrated fiber technology," Laser \& Photonics Reviews, vol. 6, no. 6, pp. 709-723, 2012.

[7] R. J. Williams, R. G. Krämer, S. Nolte, and M. J. Withford, "Femtosecond direct-writing of low-loss fiber bragg gratings using a continuous core-scanning technique," Optics letters, vol. 38, no. 11, pp. 1918-1920, 2013.

[8] R. J. Williams, N. Jovanovic, G. D. Marshall, G. N. Smith, M. J. Steel, and M. J. Withford, "Optimizing the net reflectivity of point-by-point fiber bragg gratings: the role of scattering loss," Optics Express, vol. 20, no. 12, pp. 13 451-13456, may 2012.

[9] T. Erdogan, "Fiber grating spectra," Journal of Lightwave Technology, vol. 15, no. 8, pp. 1277-1294, 1997.

[10] K. Zhou, M. Dubov, C. Mou, L. Zhang, V. K. Mezentsev, and I. Bennion, "Line-by-line fiber bragg grating made by femtosecond laser," IEEE Photonics Technology Letters, vol. 22, no. 16, pp. 1190-1192, 2010.

[11] T. A. Goebel, G. Bharathan, M. Ams, M. Heck, R. G. Krämer, C. Matzdorf, D. Richter, M. P. Siems, A. Fuerbach, and S. Nolte, "Realization of aperiodic fiber bragg gratings with ultrashort laser pulses and the line-by-line technique," Optics Letters, vol. 43, no. 15, pp. 3794 3797, aug 2018.

[12] A. Theodosiou, A. Lacraz, A. Stassis, C. Koutsides, M. Komodromos, and K. Kalli, "Plane-by-plane femtosecond laser inscription method for single-peak bragg gratings in multimode CYTOP polymer optical fiber," Journal of Lightwave Technology, vol. 35, no. 24, pp. 5404-5410, dec 2017.

[13] A. Theodosiou, A. Ioannou, and K. Kalli, "All-in-fiber cladding interferometric and bragg grating components made via plane-by-plane femtosecond laser inscription," Journal of Lightwave Technology, vol. 37, no. 18, pp. 4864-4871, sep 2019.

[14] A. Ioannou, A. Theodosiou, C. Caucheteur, and K. Kalli,
"Direct writing of plane-by-plane tilted fiber bragg gratings using a femtosecond laser," Optics letters, vol. 42, no. 24, pp. 5198-5201, 2017.

[15] P. Lu, S. J. Mihailov, H. Ding, D. Grobnic, R. B. Walker, D. Coulas, C. Hnatovsky, and A. Y. Naumov, "Plane-byplane inscription of grating structures in optical fibers," Journal of Lightwave Technology, vol. 36, no. 4, pp. 926931, 2018.

[16] R. Osellame, S. Taccheo, M. Marangoni, R. Ramponi, P. Laporta, D. Polli, S. De Silvestri, and G. Cerullo, "Femtosecond writing of active optical waveguides with astigmatically shaped beams," JOSA B, vol. 20, no. 7, pp. 1559-1567, 2003.

[17] M. Ams, G. Marshall, D. Spence, and M. Withford, "Slit beam shaping method for femtosecond laser direct-write fabrication of symmetric waveguides in bulk glasses," Optics express, vol. 13, no. 15, pp. 5676-5681, 2005.

[18] Y. Lai, Y. Chen, and M. W. Cheong, "Low-loss, high reflectivity, first-order, pitch-by-pitch fiber bragg grating fabrication in truly free-standing single-mode fiber," in Journal of Physics: Conference Series, vol. 679, no. 1. IOP Publishing, 2016, p. 012055.

[19] R. R. Thomson, A. S. Bockelt, E. Ramsay, S. Beecher, A. H. Greenaway, A. K. Kar, and D. T. Reid, "Shaping ultrafast laser inscribed optical waveguides using a deformable mirror," Optics Express, vol. 16, no. 17, pp. 12 786-12 793, aug 2008.

[20] F. He, H. Xu, Y. Cheng, J. Ni, H. Xiong, Z. Xu, K. Sugioka, and K. Midorikawa, "Fabrication of microfluidic channels with a circular cross section using spatiotemporally focused femtosecond laser pulses," Optics Letters, vol. 35, no. 7, pp. 1106-1108, mar 2010.

[21] K. J. Moh, Y. Y. Tan, X.-C. Yuan, D. K. Y. Low, and Z. L. $\mathrm{Li}$, "Influence of diffraction by a rectangular aperture on the aspect ratio of femtosecond direct-write waveguides," Optics Express, vol. 13, no. 19, pp. 7288-7297, 2005.

[22] K. Lammers, M. Siems, M. Ehrhardt, A. Alberucci, A. Szameit, H. Gross, and S. Nolte, "Nanograting based birefringent retardation elements in integrated photonic circuits," in Laser-based Micro-and Nanoprocessing XIV, U. Klotzbach, Ed. SPIE, 2020.

[23] K. Zhou, F. Shen, G. Yin, and L. Zhang, "Optical fiber micro-devices made with femtosecond laser," in Optical Sensors. OSA, 2016, pp. SeW3D-1.

[24] C. J. Bellair, C. L. Curl, B. E. Allman, P. J. Harris, A. Roberts, L. M. D. Delbridge, and K. A. Nugent, "Quantitative phase amplitude microscopy IV: imaging thick specimens," Journal of Microscopy, vol. 214, no. 1, pp. 62-69, apr 2004.

[25] M. Lancry, B. Poumellec, J. Canning, K. Cook, J. Poulin, and F. Brisset, "Ultrafast nanoporous silica formation driven by femtosecond laser irradiation," Laser \& Photonics Reviews, vol. 7, no. 6, pp. 953-962, jul 2013.

[26] C. Caucheteur, T. Guo, and J. Albert, "Polarizationassisted fiber bragg grating sensors: Tutorial and review," Journal of Lightwave Technology, vol. 35, no. 16, pp. 3311-3322, aug 2017. 